\title{
On a Characterization of Gauss Codes*
}

\author{
H. de Fraysseix and P. Ossona de Mendez \\ CNRS UMR 0017, EHESS, \\ 54 Boulevard Raspail, 75006 Paris, France \\ \{hf,pom\}@ehess.fr
}

\begin{abstract}
The traversal of a self-crossing closed plane curve, with points of multiplicity at most two, defines a double occurrence sequence, the Gauss code of the curve. Using the D-switch operation, we give a new simple characterization of these sequences and deduce a simple self-contained proof of Rosenstiehl's characterization.
\end{abstract}

\section{Introduction}

The traversal of a self-crossing closed plane curve, with points of multiplicity at most two, defines a double occurrence sequence, which we call the Gauss code of the curve, as it was first defined by Gauss [7]. While enumerating all possible codes corresponding to curves having up to five crossing points, Gauss remarked that between the two occurrences of any point there were an even number of points. This property has been formally proved by Nagy [11]. Gauss also noticed that this condition is not sufficient to characterize Gauss codes.

Lovász and Marx introduced another necessary condition [9] and eventually Rosenstiehl gave a complete combinatorial characterization [12], [16].

In the meantime, Dehn proved that the successive switching of all the points of a cross curve gives rise to a touch curve [4]. He also proved that the successive switching of all the points of a Gauss code gives rise to a double occurrence sequence having a specific bicoloration property. Unfortunately, this property is not sufficient to characterize Gauss codes. Using the D-switch, a slight modification of the switch operation, we obtain a new characterization of Gauss codes which leads to a short self-contained proof of Rosenstiehl's characterization.

For further works on Gauss codes, we refer the reader to the Bibliography.

\footnotetext{
* This work was partially supported by the Esprit LTR Project No. 20244-ALCOM IT.
} 


\section{Definitions and Notations}

\subsection{Curves and Sequences}

We first recall and introduce some definitions and notations concerning topological properties of closed plane curves.

A parametrized curve $C$ is a continuous mapping $C:[0,1] \rightarrow \mathbb{R}^{2}$, such that $C(0)=$ $C(1)$ and for which the underlying curve $C([0,1])$ is piecewise smooth and has a finite number of multiple points, all of which have multiplicity two. Let $P(C)$ denote the set of the points of multiplicity two. To any point $p \in P(C)$, we associate the two parameter values $t_{p}^{\prime}$ and $t_{p}^{\prime \prime}$, such that $t_{p}^{\prime}<t_{p}^{\prime \prime}$ and $C\left(t_{p}^{\prime}\right)=C\left(t_{p}^{\prime \prime}\right)=p$. A point $p \in P(C)$ is a crossing point if any local deformation of $C$ in a neighborhood of $t_{p}^{\prime}$ preserves the existence of a double point. Otherwise, $p$ is a touching point. A touch curve (resp. a cross curve) is a parametrized curve with only touching points (resp. crossing points).

There are two different types of touching points, depending on the local behavior of the parametrized curve:

Type 1



Type 2

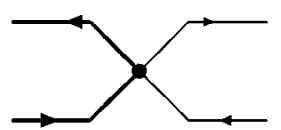

Remark. All the touch points of a touch curve are of type 1. By a local deformation we may suppress all the touch points but any given one, which then is (and was) of type 1.

The sequence of the points of $P(C)$ encountered as the parameter $t$ goes from 0 to 1 (excluded) is the traversal sequence of $C$ and is denoted by $S(C)$.

In the following, sequences are understood to have two occurrences of each symbol and to be defined up to reversal and cyclic permutation. Given a sequence $S$, two symbols $p, q$ are interlaced in $S$ if exactly one occurrence of $q$ appears in $S$ between the two occurrences of $p$ (and thus exactly one occurrence of $p$ appears in $S$ between the two occurrences of $q$ ). We denote by $\Lambda(S)$ the interlacement graph of $S$ defined by the interlacement relation in $S$.

A sequence $S$ is realized by a parametrized curve $C$ if $S$ is the traversal sequence of $C$. A sequence is touch realizable (resp. cross realizable) if it can be realized by a touch curve (resp. a cross curve). So, saying that a sequence is cross realizable means that it is a Gauss code of some self-intersecting closed curve.

\subsection{Switches and D-Switches}

We recall the switch operation [4], [8]: Given a point $p$ of $P(C)$, the curve $C^{\prime}=C \circ p$ is defined by

$$
C^{\prime}(t)= \begin{cases}C(t) & \text { if } t \notin\left[t_{p}^{\prime}, t_{p}^{\prime \prime}\right] \\ C\left(t_{p}^{\prime}+t_{p}^{\prime \prime}-t\right) & \text { if } t \in\left[t_{p}^{\prime}, t_{p}^{\prime \prime}\right]\end{cases}
$$


This curve has the same touching and crossing points as $C$, with the possible exception of $p$. The traversal sequence of $C^{\prime}$ is obtained from the one of $C$ by inverting the order of the points encountered between the two occurrences of $p$. We say that the points that are interlaced with $p$ have been inverted. The switch operation on $S$ will be denoted by $S \circ p$, so that $S(C \circ p)=S(C) \circ p$. We remark that these switch operations are involutions: $C \circ p \circ p=C$ and $S \circ p \circ p=S$.

Remark. A switch at a point $p$ transforms $p$ in the following way:

- touching point of type $1 \leftrightarrow$ crossing point,

- touching point of type $2 \leftrightarrow$ touching point of type 2 .

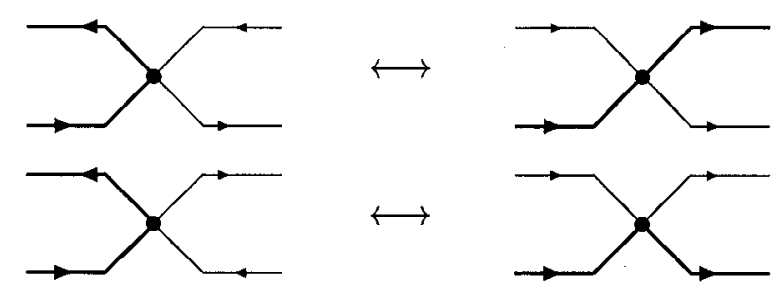

Remark. A touching point $q$ of a parametrized curve $C$ is a touching point of $C \circ p$ $(p \neq q)$ which has a different type in $C$ and $C \circ p$ if and only if $p$ and $q$ are interlaced in $S(C)$ (that is, if $q$ has been inverted by the switch at $p$ ).

Definition 1. The local complement of a graph $G$ at a vertex $v$ is the graph $G \circ v$ with the same vertex set as $G$ and the same edges as $G$ except that the neighborhood of $v$ in $G$ is complemented. Note that this is called the local complementation of $G$ in [3].

Remark. According to the definition of a switch and the definition of a local complement, we have

$$
\Lambda(S \circ p)=\Lambda(S) \circ p .
$$

This is the reason why we denote both operations by "o".

Definition 2 (D-Switch and Twin). Let $S$ be a sequence, a $D$-switch at $p$ consists of a switching at $p$ and the addition of two occurrences of a new symbol $p^{\prime}$, called the twin of $p$, one just after the first occurrence of $p$ and one just before the second occurrence of $p$. The D-switch of a sequence $S$ at a point $p$ is denoted by $S \oplus p$.

$$
S=(\alpha p \beta p \gamma) \mapsto S \oplus p=\left(\alpha p p^{\prime} \beta^{-1} p^{\prime} p \gamma\right) \text {. }
$$

Definition 3 [3]. The D-local complement of a graph $G$ at a vertex $v$ is the graph $G \oplus v$ obtained from $G \circ v$ by adding a new vertex $v^{\prime}$ having the same neighbors as $v$. 
Remark. According to the definition of a D-switch and the definition of a D-local complement, we have

$$
\Lambda(S \otimes p)=\Lambda(S) \oplus p .
$$

This is the reason why we denote both operations by "ळ".

Remark. The sequence obtained from $S \oplus p \oplus p$ by deleting the two twins of $p$ is equal to $S$.

\subsection{Remarks on Switch Operations}

We recall a result by Dehn [4] (which follows from the remarks of the previous section) and mention the major difficulties encountered when trying to use it to characterize cross realizable sequences.

Proposition 4 [4]. Consider a cross curve $C$ and any given order $\left(p_{1}, \ldots, p_{n}\right)$ of the points of $C$. Then the parametrized curve $C \circ p_{1} \circ \cdots \circ p_{n}$ obtained from $C$ by successively switching the $p_{i}$ is a touch curve.

The converse of this proposition is not true (e.g., the sequence $(a b a b)$ is not cross realizable).

Remarks. We denote by $S \stackrel{\circ}{\longrightarrow} S^{\prime}$ the existence of an order $\left(p_{1}, \ldots, p_{n}\right)$ of the symbols of $S$, such that $S^{\prime}=S \circ p_{1} \circ \cdots \circ p_{n}$.

- A cross realizable sequence does not determine the cross curve itself up to a homeomorphism.

- One may find a cross realizable sequence $S_{1}$, a noncross realizable sequence $S_{2}$, and a touch realizable sequence $S_{T}$, such that $S_{1} \stackrel{\circ}{\longrightarrow} S_{T}$ and $S_{2} \stackrel{\circ}{\longrightarrow} S_{T}$. Actually, $S_{1}$ and $S_{2}$ may be proved to have different interlacement graphs.

- Two different cross realizable sequences $S_{1}$ and $S_{2}$ may have the same interlacement graph (e.g., the sequences (abcaefdcbefd) and (acbaefdbcefd)). However, no sequence $S_{T}$ satisfies $S_{1} \stackrel{\circ}{\longrightarrow} S_{T}$ and $S_{2} \stackrel{\circ}{\longrightarrow} S_{T}$.

\section{A New Characterization of Gauss Codes}

We first state a lemma characterizing touch realizable sequences.

Lemma 5. A sequence $S$ is touch realizable if and only if its interlacement graph $\Lambda(S)$ is bipartite.

Proof. Figure 1 shows the bijection between a touch curve (I) and a bicolored chord diagram (IV):

- From I to II each touch point is split into two adjacent points with the same label, 

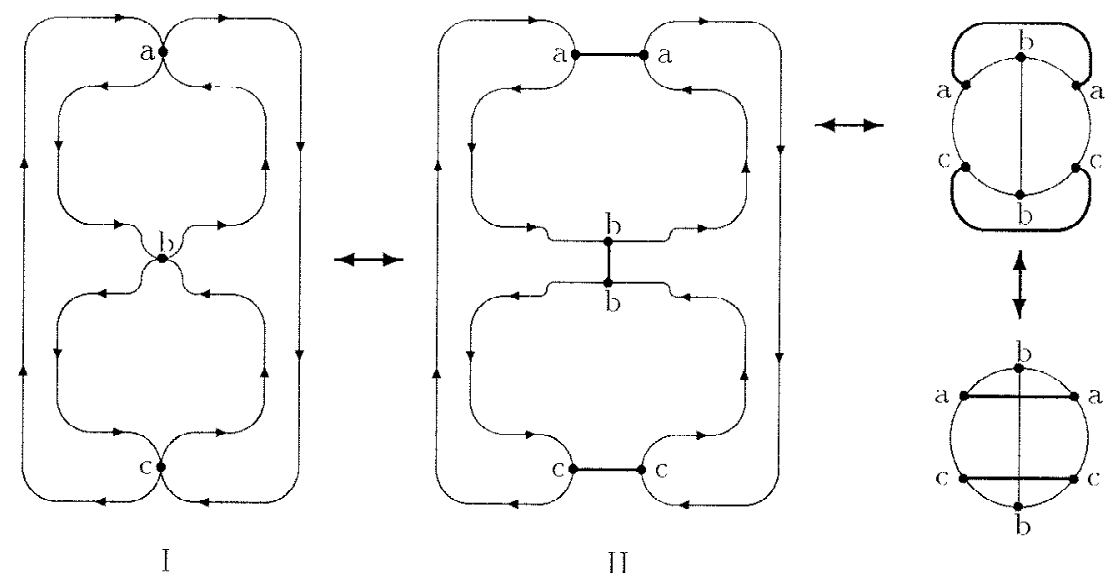

Fig. 1. Bijection between a touch curve (I) and a bicolored chord diagram (IV).

such that the traversal of the curve does not use the new edge. Conversely, II is obtained from I by contracting edges linking points with the same label.

- II and III are homeomorphic; the traversal of the original curve is now represented by a circle.

- IV is obtained from III by drawing all the chords inside the circle; the bicoloration corresponds to the inside-outside partition in III. Then two chords of the same color do not intersect. Conversely, III is obtained from IV by drawing the chords of one color outside the circle; the obtained 3-regular graph is obviously plane.

IV is a chord diagram, which is the traditional representation of the interlacement of the sequence induced by the traversal of the circle; the interlacement graph is then the intersection graph of the chords of the circle.

This bijection maps a touch curve realizing $S$ into a bicolored chord diagram representing $\Lambda(S)$, which achieves the proofs.

Theorem 6. Let $S$ be a sequence, and let $\left(p_{1}, \ldots, p_{n}\right)$ be any order on its symbols. Then $S$ is cross realizable if and only if the sequence $S_{n}=S \otimes p_{1} \otimes \cdots \oplus p_{n}$ obtained by successively $D$-switching the $p_{i}$ has a bipartite interlacement graph.

Proof. In the following, $p_{i}^{\prime}$ denotes in $S_{j}(j \geq i)$ the twin of the point $p_{i}$ introduced by the D-switch of $p_{i}$.

$(\Rightarrow)$ Assume $S$ is realized by a cross curve $C$. As a D-switch of a crossing point of a parametrized curve gives rise to two touching points (that will never become crossing points again), the curve $C$ is iteratively transformed into a touch curve $C_{n}$. The traversal sequence $S_{n}$ of $C_{n}$ has hence a bipartite interlacement graph, according to Lemma 5.

$(\Leftarrow)$ Conversely, assume that $S_{n}$ has a bipartite interlacement graph. Let $S_{i}=$ $S \otimes p_{1} \otimes \cdots \otimes p_{i}$ denote the sequence obtained after the first $i$ D-switches.

We inductively construct backward (for $i$ going from $n$ to 0 ) the parametrized curves $C_{i}$ realizing $S_{i}$, such that the crossings of $C_{i}$ are the $p_{j}$, with $j>i$. 
- Construction of $C_{n}$ :

As $\Lambda\left(S_{n}\right)$ is bipartite, there exists a touching curve $C_{n}$ whose traversal sequence is $S_{n}$.

- Construction of $C_{i-1}$ from $C_{i}(i \leq n)$ :

We prove that $p_{i}$ is always of type 1 in $C_{i}$, that is that $p_{i}$ has been inverted an even number of times during the further D-switches at $p_{i+1}, \ldots, p_{n}$ :

* The symbol $p_{i}$ and its twin $p_{i}^{\prime}$ are not interlaced in $S_{i}$,

$* p_{i}$ and $p_{i}^{\prime}$ are alternatively interlaced and not interlaced after each further inversion,

$*$ if the last inversion of $p_{i}$ occurred during a switch at $p_{j}$, then $p_{i}$ and $p_{j}$ are interlaced in $S_{n}$ and, similarly, $p_{i}^{\prime}$ and $p_{j}$ are interlaced in $S_{n}$. As $\Lambda\left(S_{n}\right)$ is bipartite, $p_{i}$ and $p_{i}^{\prime}$ are not interlaced in $S_{n}$ : otherwise $p_{i}, p_{i}^{\prime}, p_{j}$ would define a triangle of $\Lambda\left(S_{n}\right)$.

Hence, the symbol $p_{i}$ has been inverted an even number of times and $p_{i}$ is of type 1 in $C_{i}$. Hence, the suppression of $p_{i}^{\prime}$ and the switch of $p_{i}$ transforms $p_{i}$ into a crossing point and gives rise to a parametrized curve $C_{i-1}$, having $p_{i}, \ldots, p_{n}$ as crossing points and $S_{i-1}$ as a traversal sequence.

Then the parametrized curve $C_{0}$ is a cross curve realizing $S$.

Remark. A cross curve realizing the sequence $S$ could be geometrically derived from a touch curve realizing the sequence $S^{\prime}$ obtained from $S_{n}$ by suppressing all twined letters by transforming each touching point into a crossing point.

\section{Proof of Rosenstiehl's Characterization}

In [12] and [16] Rosenstiehl gave the following characterization of Gauss codes. A sequence $S$ is a Gauss code if and only if:

- $\Lambda(S)$ is Eulerian.

- For any nonedge $\{u, v\}$ of $\Lambda(S), u$ and $v$ have an even number of common vertices.

- The set of the edges $\{u, v\}$ of $\Lambda(S)$ such that $u$ and $v$ have an odd number of common vertices is a cocycle of $\Lambda(S)$.

To prove this theorem, we need a preliminary definition and two lemmas.

Definition 7. A graph $G$ together with a partition $(A, B)$ satisfies the property $P(G ; A, B)$ if any two vertices of $G$ have an odd number of common neighbors if and only if they are different, adjacent, and belong to the same class of the partition.

Remark. As a particular case, if a graph $G$ together with a partition $(A, B)$ satisfies the property $P(G ; A, B)$, then $G$ is Eulerian.

The vertex set $V$ of a graph $G$ defines the basis of a vector space $2^{V}$, with the canonical scalar product $\langle X, Y\rangle$ : the addition corresponds to the symmetric difference 
and the scalar product to the parity of the intersection. In the following, $N_{G}(u)$ denotes the neighbor set of the vertex $u$ in the graph $G$.

Lemma 8. Let $G$ be a graph with a vertex bipartition $A, B$ and let $p$ be a vertex of $G$. Let $G^{\prime}=G \oplus p$ and let $A^{\prime}, B^{\prime}$ be the vertex bipartition of $G^{\prime}$ defined by: $A^{\prime}=$ $A+N(p), B^{\prime}=B+N(p)$ and assigning $p^{\prime}$ to the class of $p$. Then $P(G ; A, B)$ implies $P\left(G^{\prime} ; A^{\prime}, B^{\prime}\right)$.

Proof. According to the definition of a D-complement, we have (in $2^{V}$ ):

- $N_{G^{\prime}}\left(p^{\prime}\right)=N_{G^{\prime}}(p)=N_{G}(p)$,

- $N_{G^{\prime}}(u)=N_{G}(u)$, if $u$ is not adjacent to $p$ (in $G$ or equivalently in $G^{\prime}$ ),

- $N_{G^{\prime}}(u)=N_{G}(u)+u+N_{G}(p)+p^{\prime}$, if $u$ is adjacent to $p$.

Let $u$ and $v$ be two vertices of $G^{\prime}$. We shall prove that the pair $(u, v)$ does not falsify $P\left(G^{\prime} ; A^{\prime}, B^{\prime}\right)$. As $p$ and $p^{\prime}$ have the same neighbors, are not adjacent, and belong to the same class in $G^{\prime}$, by considering $p$ instead of $p^{\prime}$, we may reduce to the case where neither $u$ nor $v$ is equal to $p^{\prime}$. Then we have three exclusive cases to consider:

- The vertices $u$ and $v$ are not adjacent or equal to $p$.

Then their neighborhoods, their class, and their number of common neighbors are the same in $G$ and $G^{\prime}$. Thus, the pair $u, v$ does not falsify $P\left(G^{\prime} ; A^{\prime}, B^{\prime}\right)$.

- The vertex $u$ is adjacent to $p$ and $v$ is not adjacent or equal to $p$.

$$
\begin{aligned}
\left\langle N_{G^{\prime}}(u), N_{G^{\prime}}(v)\right\rangle & =\left\langle N_{G}(u)+u+N_{G}(p)+p^{\prime}, N_{G}(v)\right\rangle \\
& =\left\langle N_{G}(u), N_{G}(v)\right\rangle+1 .
\end{aligned}
$$

As $u$ and $v$ belong to the same class $\left(A^{\prime}, B^{\prime}\right)$ if and only if they do not belong to the same class $(A, B)$ and as they are adjacent, the pair $u, v$ does not falsify $P\left(G^{\prime} ; A^{\prime}, B^{\prime}\right)$.

- The vertices $u$ and $v$ are both adjacent to $p$.

$$
\begin{aligned}
\left\langle N_{G^{\prime}}(u), N_{G^{\prime}}(v)\right\rangle & =\left\langle N_{G}(u)+u+N_{G}(p)+p^{\prime}, N_{G}(u)+v+N_{G}(p)+p^{\prime}\right\rangle \\
& =\left\langle N_{G}(u), N_{G}(v)\right\rangle+\langle N(u), N(p)\rangle+\langle N(v), N(p)\rangle+1 .
\end{aligned}
$$

As $u$ is adjacent to $p,\langle N(u), N(p)\rangle=1$ if and only if $u$ and $p$ belong to the same class $(A, B)$. So, $\langle N(u), N(p)\rangle+\langle N(v), N(p)\rangle+1=1$ if and only if $u$ and $v$ belong to the same class $(A, B)$. As $\left\langle N_{G}(u), N_{G}(v)\right\rangle=1$ if and only if $u$ and $v$ are adjacent in $G$ and belong to the same class $(A, B),\left\langle N_{G^{\prime}}(u), N_{G^{\prime}}(v)\right\rangle=1$ if and only if $u$ and $v$ are not adjacent in $G$ and belong to the same class $(A, B)$, that is, if and only if they are adjacent in $G^{\prime}$ and belong to the same class $\left(A^{\prime}, B^{\prime}\right)$. Thus, the pair $u, v$ does not falsify $P\left(G^{\prime} ; A^{\prime}, B^{\prime}\right)$.

Lemma 9. Let $G$ be a graph with a vertex bipartition $A, B$ and let $p$ be a vertex of $G$. Let $G^{\prime}=G \otimes p$ and let $A^{\prime}, B^{\prime}$ be the vertex bipartition of $G^{\prime}$ defined by $A^{\prime}=$ $A+N(p), B^{\prime}=B+N(p)$ and assigning $p^{\prime}$ to the class of $p$. Then $P\left(G^{\prime} ; A^{\prime}, B^{\prime}\right)$ implies $P(G ; A, B)$. 
Proof. Let $G^{\prime \prime}=G^{\prime} \oplus p$ and let $A^{\prime \prime}, B^{\prime \prime}$ be the vertex bipartition of $G^{\prime \prime}$ defined by $A^{\prime \prime}=A^{\prime}+N(p), B^{\prime \prime}=B^{\prime}+N(p)$, and assigning $p^{\prime \prime}$ to the class of $p$. By Lemma 8, $P\left(G^{\prime} ; A^{\prime}, B^{\prime}\right)$ implies $P\left(G^{\prime \prime} ; A^{\prime \prime}, G^{\prime \prime}\right)$. The deletion of the two twins $p^{\prime}$ and $p^{\prime \prime}$ of $p$ ensures that $P(G ; A, B)$ also holds.

Theorem 10. A sequence $S$ is a Gauss code if and only if:

$-\Lambda(S)$ is Eulerian,

- for any nonedge $\left(p, p^{\prime}\right)$ of $\Lambda(S),\left|N(p) \cap N\left(p^{\prime}\right)\right|$ is even,

- the set of the edges $\left(p, p^{\prime}\right)$ of $\Lambda(S)$ such that $\left|N(p) \cap N\left(p^{\prime}\right)\right|$ is even is a cocycle $\Lambda(S)$.

Proof. The theorem may be restated as follows: a sequence $S$ is cross realizable if and only if there exists a bipartition $A, B$ of the vertex set of $\Lambda(S)$, such that $P(\Lambda(S) ; A, B)$ holds.

$(\Leftarrow)$ Assume there exists a bipartition $A, B$ of the vertex set of $\Lambda(S)$ such that $P(\Lambda(S) ; A, B)$ holds. Consider any sequence $S^{\prime}=S \oplus p_{1} \oplus \cdots \otimes p_{n}$ obtained by successively D-switching the symbols of $S$. According to Lemma $8, \Lambda\left(S^{\prime}\right)$ has a bipartition $A^{\prime}, B^{\prime}$ such that $P\left(\Lambda\left(S^{\prime}\right) ; A^{\prime}, B^{\prime}\right)$ holds. As all the symbols have been twined and as $p$ and its twin $p^{\prime}$ have the same neighbors, any two vertices of $\Lambda\left(S^{\prime}\right)$ have an even number of common vertices. According to property $P\left(\Lambda\left(S^{\prime}\right) ; A^{\prime}, B^{\prime}\right)$, the graph $\Lambda\left(S^{\prime}\right)$ is bipartite. Then, from Theorem $6, S$ is cross realizable.

$(\Rightarrow)$ Conversely, if $S$ is cross realizable, then any sequence of D-switches gives rise to a sequence $S^{\prime}$ having a bipartite interlacement graph; any bicoloration of it defines a bipartition $A, B$ such that $P\left(\Lambda\left(S^{\prime}\right) ; A, B\right)$ holds. The theorem then follows from Lemma 9.

\section{Conclusion}

Although the characterization of double and triple occurrences which are cross realizable can be reduced to the characterization of Gauss codes [2], no characterization is known for the general case where any letter may occur any number of times.

\section{Acknowledgment}

The authors would like to thank the editor and the referee for their constructive comments and remarks.

\section{Bibliography}

1. A. Bouchet. Caractérisation des symboles croisés de genre nul. C. R. Acad. Sci. Paris, 274:724-727, 1972.

2. H. de Fraysseix. Sur la représentation d'une suite à triples et à doubles occurrences par la suite des points d'intersection d'une courbe fermée du plan. In Problèmes combinatoires et théorie des graphes, volume 260 of Colloques internationaux C.N.R.S., pages 161-165. C.N.R.S., Paris, 1976. 
3. H. de Fraysseix. Local complementation and interlacement graphs. Discrete Math., 33:29-35, 1981.

4. M. Dehn. Über kombinatorische Topologie. Acta Math., 67:123-168, 1936.

5. H. Fleischner. Cycle decompositions, 2-coverings, removable cycles, and the four-color-disease. Progress in Graph Theory, pages 233-246, 1984.

6. G.K. Francis. Null genus realizability criterion for abstract intersection sequences. J. Combin. Theory, 7:331-341, 1969.

7. C.F. Gauss. Werke, pages 272 and 282-28. Teubner, Leipzig, 1900.

8. A. Kotzig. Eulerian lines in finite 4-valent graphs and their transformations. In Proceedings of the Colloquium held at Tihany, Hungary, pages 219-230, 1969.

9. L. Lovász and M.L. Marx. A forbidden subgraph characterization of gauss codes. Bull. Amer. Math. Soc., 82:121-122, 1976.

10. M.L. Marx. The gauss realizability problem. Proc. Amer. Math. Soc., 22:610-613, 1969.

11. J.V.Sz. Nagy. Über ein topologisches Problem von Gauss. Math. Z., 26:579-592, 1927.

12. R.C. Read and P. Rosenstiehl. On the Gauss Crossing Problem. Colloquia Mathematica Societatis János Bolyai, pages 843-875. North-Holland, Amsterdam, 1976.

13. R.C. Read and P. Rosenstiehl. On the principal edge tripartition of a graph. Ann. Discrete Math., 3:195-226, 1978.

14. P. Rosenstiehl. A new proof of the gauss interlace conjecture. (In preparation.)

15. P. Rosenstiehl. Les graphes d'entrelacement d'un graphe. In Problèmes combinatoires et théorie des graphes, volume 260 of Colloques internationaux C.N.R.S., pages 359-362. C.N.R.S., 1976.

16. P. Rosenstiehl. Solution algebrique du problème de Gauss sur la permutation des points d'intersection d'une ou plusieurs courbes fermées du plan. C. R. Acad. Sci. Paris, 283(A):551-553, 1976.

17. P. Rosenstiehl and R.E. Tarjan. Gauss codes, planar hamiltonian graphs, and stack-sortable permutations. Algorithms, 5:375-390, 1984.

18. H. Shank. The theory of left-right paths. In Combinatorial Mathematics, volume III of Lecture Notes in Mathematics, pages 42-54. Springer-Verlag, Berlin, 1975.

19. L.B. Treybig. A characterization of the double point structure of a projection of a polygonal knot in regular position. Trans. Amer. Math. Soc., 30:223-247, 1968.

20. W.T. Tutte. On unicursal paths in a network of degree 4. Amer. Math. Monthly, 4:233-237, 1941.

Received October 13, 1997, and in revised form March 29, 1998. 